\title{
An investigation on the presence of Chlamydiaceae in Swedish dogs
}

Bodil Ström Holst ${ }^{1,2^{*}}$, Sofia Hanås ${ }^{3}$, Göran Bölske ${ }^{1}$, Catharina Linde Forsberg ${ }^{2}$

\begin{abstract}
Background: Bacteria belonging to the family Chlamydiaceae cause a broad spectrum of diseases in a wide range of hosts, including man, other mammals, and birds. Upper respiratory and genital diseases are common clinical problems caused by Chlamydiaceae. Very little is known about chlamydial infections in dogs. Few clinical reports on natural disease in dogs describe mainly conjunctival and upper respiratory signs, and the role of Chlamydiaceae in genital disease is unclear. The present study aimed at studying the prevalence of Chlamydiaceae in healthy dogs and in dogs with genital or upper respiratory disease, including conjunctivitis.

Methods: A real-time polymerase chain reaction (PCR) for Chlamydiaceae was used to detect any chlamydial species within this family. Swab samples from the conjunctiva and the mucosal membranes of the oropharynx, rectum and genital tract were taken from 79 dogs: 27 clinically healthy dogs, 25 dogs with clinical signs from the genital tract and 28 dogs with conjunctivitis. There were 52 female and 27 male dogs. From 7 of the male dogs, additional semen samples were analysed.

Results: No Chlamydiaceae were detected from any dog.

Conclusions: Although the number of dogs that was included is limited, the results suggest that cases of Chlamydiaceae in dogs probably are related to infection from other species, and that dogs in general do not harbour Chlamydiaceae. Bacteria belonging to the family Chlamydiaceae do not seem to be of major importance for genital or ocular disease in Swedish dogs.
\end{abstract}

\section{Background}

Chlamydiae are obligately intracellular bacteria belonging to the bacterial order Chlamydiales. The Chlamydiales comprises four families: Chlamydiaceae, Parachlamydiaceae, Simkaniaceae, and Waddliaceae. The family Chlamydiaceae contains two genera: Chlamydia and Chlamydophila [1]. Bacteria belonging to the family Chlamydiaceae cause a broad spectrum of diseases in a wide range of hosts, including man, other mammals, and birds. Reproductive disturbances are common, among them salpingitis in man (caused by Chlamydia trachomatis), genitourinary disease in koalas (caused mainly by Chlamydophila pecorum) and abortions in sheep and cattle (caused mainly by Chlamydophila abortus) [2,3]. In cats there is also circumstantial evidence for a role of Chlamydiaceae in reproductive disturbances, and chlamydiae have been isolated from

\footnotetext{
* Correspondence: Bodil.Strom-Holst@kv.slu.se

${ }^{1}$ National Veterinary Institute (SVA), Uppsala, Sweden

Full list of author information is available at the end of the article
}

the vagina of cats, e.g. [4]. Bacteria belonging to the family Chlamydiaceae are also common ocular pathogens, for instance in humans (C. trachomatis), koalas (Cp. pecorum) and cats (Chlamydophila felis). Avian chlamydiosis, caused by Chlamydophila psittaci, occurs worldwide and $C p$. psittaci has been detected from a wide variety of both wild and domesticated birds. Avian chlamydiosis is the most important animal chlamydiosis transmissible to man (causing respiratory disease; ornithosis or psittacosis), but other chlamydiae, such as $C p$. abortus and $C p$. felis, also have a zoonotic potential [3], although $C p$. felis infection in humans hitherto has been verified in only one, HIV-positive, case [5].

Very little is known regarding the potential of Chlamydiaceae to cause genital or upper respiratory disease including ocular disease in dogs. Detection of Chlamydiaceae requires special cultivation techniques, or analysis with polymerase chain reaction (PCR), and therefore these bacteria are seldom searched for in clinical cases of genital disease or reproductive disturbances. Other 
aetiological agents, such as canine herpes virus or extracellular aerobic bacteria, that are known to have the capacity of causing genital disease and reproductive problems in dogs, are searched for more frequently. Many cases of conjunctivitis are also treated on an empirical basis, without attempts to make an etiological diagnosis, and if a causative agent is searched for, this is rarely chlamydiae.

The aim of the present study was to study if members of the family Chlamydiaceae can be detected on the mucosa of healthy dogs, from dogs with reproductive disturbances or genital disease, or from dogs with upper respiratory signs including conjunctivitis. A real-time PCR that detects all members of the family Chlamydiaceae was used. No Chlamydiaceae was detected from any of the 4-5 sampling sites from the 79 dogs in the present study.

\section{Methods}

\section{Ethical approval}

The study was approved by the Uppsala ethical committee for animal research (C 310/05) and by the Swedish animal welfare agency (2006-0047). All dog owners gave their informed written consent.

\section{Dogs}

79 privately own dogs of 41 different breeds, five mixed breeds and 5 with unknown breed, were included in the study. There were 52 females and 27 males. They were categorized as clinically healthy $(\mathrm{N}=27)$, with clinical symptoms from the genital tract $(\mathrm{N}=25)$, or from the upper respiratory tract, including ocular signs $(\mathrm{N}=28)$. One bitch was sampled twice, first with cystic endometrial hyperplasia $(\mathrm{CEH}) /$ endometritis and 2 years later with conjunctivitis.

Signs from the genital tract included $\mathrm{CEH} /$ endometritis or vaginal discharge $(\mathrm{N}=12)$, pyometra $(\mathrm{N}=6)$, vulvar lesions $(\mathrm{N}=1)$, poor semen quality $(\mathrm{N}=1)$, failure to conceive $(\mathrm{N}=1)$, prostatic disease $(\mathrm{N}=1)$, aberrant oestrous cycles $(\mathrm{N}=2)$ and balanoposthitis $(\mathrm{N}=1)$. Upper respiratory signs included conjunctivitis $(\mathrm{N}=20)$, conjunctivitis and cough or sneezing $(\mathrm{N}=4)$, and only cough or sneezing $(\mathrm{N}=4)$. The dogs were sampled with cotton tipped swabs without any transport or culture medium. From each dog, four samples were taken: from the conjunctiva, oropharynx, rectum and genital tract (vagina or preputium) by rolling swabs against the mucosa. From male dogs, an additional fifth swab was dipped in semen samples, when such a sample was available $(\mathrm{N}=7)$.

\section{Real-time PCR analysis}

At the laboratory, DNA was extracted from the swabs for PCR analysis according to the protocol by Sachse and Hotzel [6]. The samples were analysed using a real-time PCR originally developed by Everett et al. [2], which targets the $23 \mathrm{~S}$ ribosomal DNA and detects all members of the family Chlamydiaceae. We have modified this real-time PCR and developed an internal control to monitor any inhibition from the sample material [7].

\section{Results and discussion}

Seventeen of the bitches (22\%) were in heat and seventeen were in metoestrus. Of the 79 dogs, 27 (34\%) were stud dogs or breeding bitches. For 24 dogs $(30 \%)$, previous reproductive disturbances or genital disease was reported, and 26 dogs (33\%) had previously had conjunctivitis. No Chlamydiaceae were detected in any sample.

The present study aimed at studying if healthy dogs or dogs with genital or upper respiratory including ocular disease harboured chlamydiae on their mucosa. In chronically infected ewes, the periovulatory period has been described to be optimal for detecting chlamydiae [8]. To increase the possibility of detecting chlamydiae in possibly infected bitches, several bitches (22\%) were sampled in heat. Despite this, Chlamydiaceae were not detected from any of the samples.

Few seroepidemiological studies on chlamydiae in dogs have been performed. In a Japanese study, using a complement fixation test, $9.5 \%$ of dogs were seropositive for $C p$. psittaci, which was an even higher number than for the cats in the same study [9]. There was also a large variation in seropositivity between different dog populations, from 0.9 to 17.4 per cent, with the highest seroprevalence in a colony of experimental animals. In a German study, using an enzyme-linked immunosorbent assay (ELISA), 20\% of the dogs were seropositive for $C p$. psittaci: $19.8 \%$ in a population of healthy dogs $(\mathrm{N}=$ 1028 ) and $26 \%$ of dogs with various clinical signs $(\mathrm{N}=$ 99) [10]. These results would indicate that infection with chlamydiae is not unusual in dogs. However, reports on naturally occurring chlamydial disease in dogs are scarce. Conjunctivitis caused by $C p$. abortus has been described [11]. The source of infection could not be traced, but as the dog was living on the countryside, there were many possible infection routes directly and indirectly from sheep [11]. Coughing, dyspnoea, oculonasal discharge, pyrexia and anorexia caused by $C p$. psittaci have been described: two out of three dogs in a household were infected, probably from a cockatiel [12]. The humans also developed severe clinical signs [12]. Cp. psittaci was also detected in a sample from a dog from a colony of dogs experiencing recurrent periods of dyspnoea, keratoconjunctivitis and small litter sizes and that had been kept together with a parrot and two canary birds [13]. Humans from the same 
household also had recurrent respiratory disease [13]. From one clinical sample from a dog with unknown clinical signs, $C p$. caviae has been detected, a common chlamydial species in rabbits and guinea pigs, and from samples from 4 dogs with conjunctivitis, $C p$. felis was detected [14].

A causative relationship between chlamydial infection and genital tract disease has not been reported in dogs, although small litter sizes were reported in a colony of dogs in which $C p$. psittaci was detected in a sample from one of the dogs [13]. In an experimental setting, inflammatory changes in the prostatic gland have been induced by injecting C. trachomatis directly into the prostatic gland [15]. The dogs ceased to excrete chlamydiae after 3-16 days and no chlamydiae could be grown in tissue cultures, suggesting that the infection may have been self-limiting. No case of naturally occurring prostatic disease due to chlamydiae in dogs has been described, and no Chlamydiaceae were detected from the dog with prostatic disease in the present study. Although it has been suggested that the dog might be a model for chlamydial prostatitis [15] it does not seem likely that chlamydiae are natural causes of canine prostatic disease. Based on the scarcity of clinical reports, and the lack of naturally occurring chlamydiae on the canine mucosa found in the present study, chlamydial infection is likely an uncommon cause of reproductive disturbances in the canine species

Other clinical signs experienced in dogs infected with chlamydiae include hepatitis after experimental infection [16], and a suspicion of septic polyarthritis, although chlamydiae could not be cultured [17]. The organism could, however, be isolated from another dog with pyrexia and shifting leg lameness [18]. Chlamydiae have been isolated from pleural effusions of a dog referred to a clinic for pyrexia and shifting leg lameness. The chlamydiae were classified as "turkey strain" of Cp. psittaci, and it was speculated that the dog had been infected by an avian host [18].

Contact with another animal species infected with chlamydiae is thus almost always present when clinical disease caused by chlamydiae is present in dogs. Infection with chlamydiae is a potential differential etiological diagnosis in cases of especially upper respiratory disease in dogs with bird contact, and in cases of conjunctivitis after contact with cats (and maybe guinea pigs) with clinical chlamydiosis.

No case of suspected transmission of chlamydial infection between dogs has been reported. Chronic infection in dogs has been suggested, due to the identification of $C p$. pneumoniae by PCR and immunohistochemistry from atherosclerotic lesions of aortas, coronary and splenic arteries of dogs [19]. As no chlamydiae were detected in the control animals, it was suggested that
$C p$. pneumoniae may play an important role in the development of canine atherosclerotic lesions [19]. Such changes are, however, uncommon in dogs. Cp. pneumoniae was neither detected in samples from dogs with idiopathic pericardial effusions [20], nor in samples from dogs with idiopathic lymphoplasmacytic rhinitis [21]. It has also been suggested that dogs may be potential reservoirs for $C p$. psittaci, due to the persistent nature of the infection in a colony of dogs [13].

In Sweden it is required by law to report cases of ornithosis in humans to government authorities. The number of cases has decreased the last decennium, and since 2003 less than ten cases per year are reported http://www.smittskyddsinstitutet.se. The major source of infection is thought to be psittacine and other birds. Psittacosis in birds is also notifiable in Sweden, the number of cases being less than ten per year http:// www.sjv.se. In Swedish dairy cows, Cp. abortus is absent or the prevalence is very low whereas $C p$. pecorum was identified by PCR from vaginal swabs from $2 / 12$ animals (17\%) [22]. The prevalence of Cp. psittaci in faeces of passerine birds in Sweden is relatively low, around 3\% $(9 / 312,[23])$. On the other hand, Chlamydiaceae is, in Sweden as in many other countries, commonly detected in samples from cats with conjunctivitis [24]. The risk for a dog to catch an infection with chlamydiae from sheep, cattle, or birds in Sweden is therefore rather low, whereas the risk of getting infected by a cat may be higher, although no such case was found in the present study.

\section{Conclusions}

No Chlamydiaceae were detected from dogs in the present study. Although the number of dogs was limited, the results suggest that single cases of Chlamydiaceae in dogs are probably related to infection from other species, and that dogs in general do not harbour Chlamydiaceae. The frequency with which chlamydial infections can be diagnosed in dogs within a certain area can thus be expected to be dependent on the prevalence in other animal species, and the frequency of contact between dogs and these infected animals. Bacteria belonging to the family Chlamydiaceae do not seem to be of major importance for ocular or genital disease in Swedish dogs. Chlamydial infection could however be considered a potential differential diagnosis in dogs with upper respiratory disease and bird contacts, or with conjunctivitis and contact with cats.

\section{Acknowledgements}

The technical staff at the Chlamydia laboratory of the National Veterinary Institute is thanked for analysis of the samples. The study was financially supported by the AGRIA Insurance Company and the Swedish Kennel Club Research fund. 


\section{Author details}

${ }^{1}$ National Veterinary Institute (SVA), Uppsala, Sweden. ${ }^{2}$ Swedish University of Agricultural Sciences (SLU), Faculty of Veterinary Medicine and Animal Health, Department of Clinical Sciences, Uppsala, Sweden. ${ }^{3}$ Animal Clinic Djurdoktorn, Västerås, Sweden.

\section{Authors' contributions}

BSH designed and coordinated the study and had the major responsibility for writing and finalising the manuscript. SH, CLF and BSH collected the samples and GB was responsible for the PCR analysis. All authors participated actively in the writing of the manuscript, and all read and approved the final manuscript.

\section{Competing interests}

The authors declare that they have no competing interests.

Received: 24 June 2010 Accepted: 16 November 2010

Published: 16 November 2010

\section{References}

1. Everett KD: Chlamydia and Chlamydiales: more than meets the eye. Vet Microbiol 2000, 75(2):109-126.

2. Everett $K D$, Hornung $L$, Andersen $A A$ : Rapid detection of the Chlamydiaceae and other families in the order Chlamydiales: three PCR tests. J Clin Microbiol 1999, 37(3):575-580.

3. Longbottom D, Coulter L: Animal chlamydioses and zoonotic implications. J Comp Pathol 2003, 128(4):217-244.

4. Sykes JE, Studdert VP, Browning GF: Comparison of the polymerase chain reaction and culture for the detection of feline Chlamydia psittaci in untreated and doxycycline-treated experimentally infected cats. J Vet Intern Med 1999, 13(3):146-152.

5. Hartley JC, Stevenson S, Robinson AJ, Littlewood JD, Carder C, Cartledge J, Clark C, Ridgway GL: Conjunctivitis due to Chlamydophila felis (Chlamydia psittaci feline pneumonitis agent) acquired from a cat: case report with molecular characterization of isolates from the patient and cat. J Infect 2001, 43(1):7-11.

6. Sachse K, Hotzel H: Detection and differentiation of Chlamydiae by nested PCR. Methods Mol Biol 2003, 216:123-136.

7. Ström Holst B, Krook L, Englund S, Lagerstedt A-S, Bölske G: Excretion of chlamydiae from clinically healthy pedigree cats. American Journal of Veterinary Research.

8. Papp JR, Shewen PE, Thorn CE, Andersen AA: Immunocytologic detection of Chlamydia psittaci from cervical and vaginal samples of chronically infected ewes. Can J Vet Res 1998, 62(1):72-74.

9. Fukushi H, Ogawa H, Minamoto N, Hashimoto A, Yagami K, Tamura H, Shimakura S, Hirai K: Seroepidemiological surveillance of Chlamydia psittaci in cats and dogs in Japan. Vet Rec 1985, 117(19):503-504.

10. Werth D, Schmeer N, Muller HP, Karo M, Krauss H: [Demonstration of antibodies against Chlamydia psittaci and Coxiella burnetii in dogs and cats: comparison of the enzyme immunoassay, immunoperoxidase technic, complement fixation test and agar gel precipitation test]. Zentralbl Veterinarmed B 1987, 34(3):165-176.

11. Hoelzle K, Wittenbrink MM, Corboz L, Hoelzle LE: Chlamydophila abortusinduced keratoconjunctivitis in a dog. Vet Rec 2005, 157(20):632-633.

12. Gresham AC, Dixon CE, Bevan BJ: Domiciliary outbreak of psittacosis in dogs: potential for zoonotic infection. Vet Rec 1996, 138(25):622-623.

13. Spraque LD, Schubert E, Hotzel H, Scharf S, Sachse K: The detection of Chlamydophila psittaci genotype C infection in dogs. Vet J 2009, 181(3):274-279.

14. Pantchev A, Sting R, Bauerfeind R, Tyczka J, Sachse K: Detection of all Chlamydophila and Chlamydia spp. of veterinary interest using speciesspecific real-time PCR assays. Comp Immunol Microbiol Infect Dis 2009.

15. Nielsen OS, Golubjatnikov R, Dodge R, Madsen PO: Chlamydial prostatitis in dogs: an experimental study. Urol Res 1982, 10(1):45-49.

16. Young S, Storz J, Maierhofer CA: Pathologic features of experimentally induced chlamydial infection in dogs. Am J Vet Res 1972, 33(2):377-383.

17. Lambrechts N, Picard J, Tustin RC: Chlamydia-induced septic polyarthritis in a dog. J S Afr Vet Assoc 1999, 70(1):40-42.

18. Arizmendi F, Grimes JE, Relford RL: Isolation of Chlamydia psittaci from pleural effusion in a dog. J Vet Diagn Invest 1992, 4(4):460-463.
19. Sako T, Takahashi T, Takehana K, Uchida E, Nakade T, Umemura T, Taniyama H: Chlamydial infection in canine atherosclerotic lesions. Atherosclerosis 2002, 162(2):253-259.

20. Zini E, Glaus TM, Bussadori C, Borgarelli M, Santilli RA, Tarducci A, Margiocco ML, Rampazzo A, Meli ML, Maisch B, et al: Evaluation of the presence of selected viral and bacterial nucleic acids in pericardial samples from dogs with or without idiopathic pericardial effusion. Vet $J$ 2009, 179(2):225-229

21. Windsor RC, Johnson LR, Sykes JE, Drazenovich $T L$, Leutenegger $C M$, De Cock HE: Molecular detection of microbes in nasal tissue of dogs with idiopathic lymphoplasmacytic rhinitis. J Vet Intern Med 2006 , 20(2):250-256.

22. Godin AC, Bjorkman C, Englund S, Johansson KE, Niskanen R, Alenius S: Investigation of Chlamydophila spp. in dairy cows with reproductive disorders. Acta Vet Scand 2008, 50:39.

23. Olsen B, Persson K, Broholm KA: PCR detection of Chlamydia psittaci in faecal samples from passerine birds in Sweden. Epidemiol Infect 1998, 121(2):481-484.

24. Holst BS, Hanas S, Berndtsson LT, Hansson I, Soderlund R, Aspan A, SjodahlEssen T, Bolske G, Greko C: Infectious causes for feline upper respiratory tract disease-a case-control study. J Feline Med Surg 2010, 12(10):783-789.

doi:10.1186/1751-0147-52-63

Cite this article as: Holst et al:: An investigation on the presence of Chlamydiaceae in Swedish dogs. Acta Veterinaria Scandinavica 2010 52:63.

\section{Submit your next manuscript to BioMed Central and take full advantage of:}

- Convenient online submission

- Thorough peer review

- No space constraints or color figure charges

- Immediate publication on acceptance

- Inclusion in PubMed, CAS, Scopus and Google Scholar

- Research which is freely available for redistribution 